\title{
Embedded Fault Diagnosis Expert System Based on CLIPS and ANN
}

\author{
Tan Dapeng, Li Peiyu, and Pan Xiaohong \\ College of Mechanical and Energy Engineering, Zhejiang University, \\ Hangzhou 310027, China \\ \{tandapeng, lipeiyu,pan_xh\}@zju.edu.cn
}

\begin{abstract}
Embedded fault diagnosis technology requires high pertinency and small occupation space. Traditional fault diagnosis system can not satisfy the demand above. Aiming at this problem, a kind of embedded fault diagnosis expert system (E-FDES) based on CLIPS and ANN was brought forward. FDES and its relative technology were discussed, developing environment and design tool chain for E-FDES was established in Linux environment. Using modularization theory principle, system re-configuration and expansion ability were guaranteed, and data process components and graphic user interface components could be reduced to meet the embedded system running requirement. By application program interface of CLIPS and Protégé plug-in, knowledge base and rule base were realized, that decreased the developing time of system inference engine and reduced source occupation space. Industry experiments prove that this system runs stably in ARM-Linux embedded environment, switch smoothly and can recognize some common faults for the devices monitored.
\end{abstract}

Keywords: embedded system, fault diagnosis, expert system, CLIPS, ANN.

\section{Introduction}

Expert system is an important research production of artificial intelligence, it can work efficiently in a small range field with difficult problems, and achieve the expert's ability. But the traditional expert system has heavy architecture, requires large scale data and space resource to keep its normal work, that can not adapt the embedded system environment demand such as small occupation space, high realtime performance, good configuration ability and so on. Aiming at the problem above, this paper introduces embedded system technology into the design process of FDES, make expert system applied in the embedded system environment[1].

\section{System Architecture Design}

According to the characteristic and special demand of FDES in embedded system environment, the developing chain of $C+C L I P S+$ Protégé $+E c l i p s e+Q t$ was adopted combined developing production and experience of relative depending project[2]. The realization method of FDES was described as follows: Firstly, bottom layer data 
support component are realized by basic language $\mathrm{C} / \mathrm{C}++$, it will finish the work of real-time data collection, pre-process, calculation and extraction of symptom data etc; Then, system inference engine frame is established around by the $\mathrm{C}$ language integration product system (CLIPS), the management and development interactivity ability of monitoring objects system is strengthened by using Protégé, a visual ontology edit tool, and the process decision and inference are realized combined artificial neural network (ANN) technology; At last, graphic user interface (GUI) component is designed by mature graphic tool library Qt/embedded, and the application instance is developed integrated by and integrated developing environment tool-Eclipse.

\section{System Crucial Technologies Design}

\subsection{Object Ontology System Based on CLIPS and Protégé}

CLIPS was developed by NASA as an expert system developing tool, it has such advantages as convenience, low cost and easy to be integrated by outer system. Protégé is an open source ontology edit tool originated by Stanford university, it is a good choice to realize the building and ontology description of detecting target system and its components. According to the technical and running character of CLIPS, object ontology management system oriented to E-FDES is designed with followed steps: 1) Every part of object system (factory, workshop, devices, sensors etc.) will be defined in the same mode, confirmed its class inheriting relation, instance and slot attribution in Protégé system frame; 2) Using the CLIPS plug-in interface of Protégé, the defined ontology system is saved as the file with "clp" expansion name, and leaded into CLIPS frame to finish the ontology realization for embedded environment. 3) In allusion to special requirement and application condition, CLIPS can modify the ontology file, then write back to Protégé frame. The above method integrates the merits of CLIPS and Protégé, resolves poor interactivity problem of CLIPS, reduces the developing time.

\subsection{Inference Engine Based on CLIPS and ANN}

Design of inference engine is the most important part of whole system realization. This paper adopts CLIPS and ANN to realize the diagnosis information integration, and obtain the diagnosis outcome. CLIPS can do fault inference by production rule, and has good rapidity. What's more, CLIPS has the ability of object-oriented, not only can realize the simply relational inference, but can process the diagnosis and recognition of complex interactive reticular fault network[3].

The application scope of expert system is very narrow, so E-FDES requires high specialty because of the space and calculation ability limitation of embedded environment. In the hand of inference effectivity, E-FDES requires long time industry fields train aiming at some kind of devices. Since ANN technology has the advantages for knowledge study, rule training and inference for random problem, this paper adopts it to realize the work above, and obtain the diagnosis outcome using information integration algorithm. ANN information integration algorithm combines the BP network and fuzzy set, then constructs a fuzzy neural network classifier, its input and output are confidence degree value with some semantic attribution. In the process of 
diagnosis, the fault symptom data from RTDCS is looked as the input of BP network, the output is the confidence value of some kind of fault, then the deep decision is done according to relative decision rule, such as fault position, fault reason and fault measure, that require several ANN to work together, and the confidence degree is obtained by confidence function[4].

The running mechanism of system inference engine is shown in Fig.1. After system start, the monitoring object ontology and its corresponding instances are awaked, then the system parameters and diagnosis threshold are set according to special application condition, and the fault symptom data from RTDCS is transferred to match with CLIPS rules. If the matching confidence degree reach or exceed the diagnosis threshold, the diagnosis outcome can be determined directly; on the contrary, based on the matching outcome, normalization information integration is done combined ANN calculation outcome, and their confidence degree is compared to obtain the final diagnosis outcome.

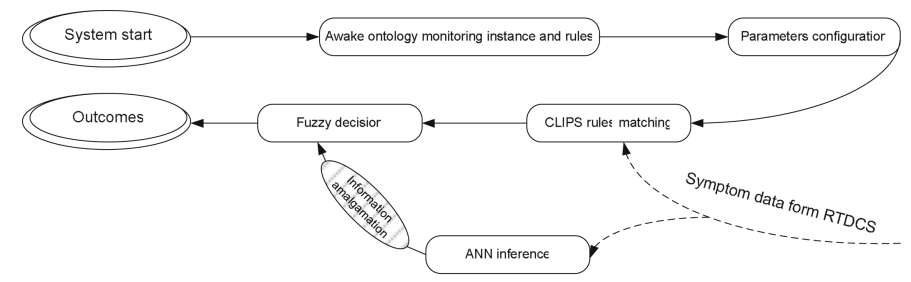

Fig. 1. System inference engine running mechanism

\section{Application Instance Experiments}

According to design steps and realization strategy, this paper has realized the E-FDES application system oriented to large-sized rotate machine. It can run stably in handheld fault diagnosis instrument self-developed, and do large number of industry field experiments in some relative factory. As shown in Fig.2, operators are detecting the pour polymer pump. The pump requires long time continuous work, its key components valve apt to occur two kinds of typical fault: spring abruption (Fault A) and surface pit corrosion (Fault B). These faults occur at high randomness, their position is hard to judge. So the pump can but demount and inspect components one by one to find the fault diagnosis. By favor of operators, this system detected some devices which occur fault frequently, it can reach the recognition ratio of ninety percent, and can find the fault position correctly, the experiments outcome detail is shown in Fig.2.
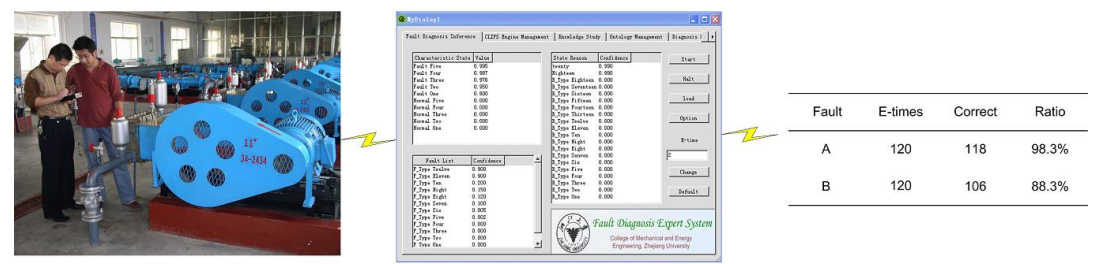

Fig. 2. Industry field application experiments 


\section{Conclusion}

This paper introduces embedded system technology into the design process of FDES. On the base of stable and quick real-time data collect module, Protégé and CLIPS's advantage are combined to reduce the developing time and strengthen the system interactivity. By the integration of CLIPS and ANN, compact and effective inference engine is realized, and system application instance is developed by Eclipse, it can adapt the embedded system environment well. The industry experiment has proved its effectivity, and the future research work will be carried out around by real-time performance optimization and GUI rapidity.

\section{References}

1. Ranganathan, S., Beetner, D. G., and Wiese, R. H.: An expert system architecture to detect system-level automotive EMC problems. In Proceedings of IEEE International Symposium on Electromagnetic Compatibility (2002).

2. Vegh, J.: The "carbon contamination" rule set implemented in an "embedded expert system". Journal of Electron Spectroscopy and Related Phenomena, vol. 133, (2003) 87-101.

3. Guo, J., Liao, Y. H., and Pamula, R.: Extending eclipse to support object-oriented system verification. In Proceedings of IEEE International Conference on Information Reuse and Integration (2005).

4. Mweene, H. V.: Confessions of an eclipse consultant. Physics World, vol. 16, (2003) 56-59. 